\title{
Arbor
}

\section{Tendencias de la Educación universitaria en el siglo XXI}

\section{Vicente Ortega Castro}

Arbor CLXXIII, 681 (Septiembre 2002), 67-81 pp.

El cambio de milenio ha propiciado muchos informes, libros y conferencias sobre la situación de las Universidades y los posibles cambios que se avecinaban como consecuencia de las tendencias de cambios en la sociedad, entre los que se encuentran el predominio del conocimiento, la globalización y la influencia de nuevas tecnologías aplicadas al ámbito de la educación. Para la sociedad europea, en la que España está inmersa política, social y económicamente, es especialmente relevante la expectativa que está despertando la Declaración de Bolonia. Pues bien, tomando este documento como referencia principal, este artículo examina las funciones permanentes de la Universidad: creación de conocimientos, formación de profesionales, servicio a la sociedad y otros, teniendo en cuenta la importancia de la calidad, de la internacionalización, de la competitividad, de la movilidad, de la complejidad, de las nuevas demandas, de los nuevos esquemas de financiación y otros factores que hacen necesario que las Universidades comprendan las nuevas tendencia y hagan un esfuerzo, no solo de adaptación, sino de anticipación.

El final del siglo XX ha sido pródigo en declaraciones e informes sobre el papel que deben jugar en el futuro las instituciones de enseñanza superior, entre las que se encuentran en lugar destacado las Universidades, sobre todo en España donde, prácticamente, toda la enseñanza superior es universitaria. Dejando aparte los artículos en revistas especializadas sobre educación superior o libros escritos por profesores de 
universidad sobre el futuro de la misma, que son muy numerosos, lo destacable es que desde instancias políticas y empresariales se han promovido y publicado informes en los que la sociedad no estrictamente universitaria ha tratado de ir delineando sus propuestas para el futuro de la enseñanza superior a la vista de los cambios sociales y económicos que se están produciendo en la sociedad y siempre con dos ideas fundamentales latentes: que la educación superior es un factor de primordial importancia para el desarrollo de la sociedad del conocimiento y que, por lo tanto, las instituciones encargadas de la enseñanza, la investigación y la formación de profesionales deben estar atentos a las necesidades de dicha sociedad, no vivir de espaldas a ellas y por lo tanto deben ser innovadoras en sus tareas y en su organización. También las Universidades han hecho sentir su voz casi siempre con más énfasis en la defensa de su autonomía institucional.

El 18 de septiembre de 1988 con motivo del noveno centenario de la creación de la Universidad de Bolonia, la más antigua de Europa y del mundo, numerosos Rectores de Universidades firmaron la «Magna Charta Universitatum». En 1996 la UNESCO publicó el llamado Informe Delors: «La Educación encierra un tesoro. Informe para la UNESCO de la Comisión Internacional sobre Educación para el siglo XXI». En julio de 1997, el Comité Nacional de Evaluación de la Educación Superior de Gran Bretaña a instancias de los Secretarios de Estado de Educación y Empleo de Gales, Escocia, Irlanda del Norte e Inglaterra publica el informe «La Educación Superior en la Sociedad del Aprendizaje», informe mas conocido como Informe Dearing. En mayo de 1998 y, por encargo del Ministro de Educación de Francia, Mr. Claude Allegre, se publica el informe "Por un Modelo Europeo de Enseñanza Superior», conocido como Informe Attali por ser Jacques Attali el presidente de una Comisión constituida. En mayo de 1999, 30 Ministros y Secretarios de Estado de 30 países europeos firman la «Declaración de Bolonia», relativa a la promoción de un Espacio Europeo de Enseñanza Superior. En España, siguiendo la moda europea, la Conferencia de Rectores de las Universidades Españolas, encarga un informe a un Comité de expertos, presidido por Josep $\mathrm{M}^{\mathrm{a}}$ Bricall, informe que es publicado con el título Universidad 2000 y que pretendía ser un documento para el debate con vistas a una futura Ley de Universidades que se presumía próxima.

Los citados anteriormente, son algunos de los documentos más significativos publicados en el ámbito europeo. Supongo que en otras regiones del mundo se habrán producido debates e informes de naturaleza parecida, como el denominado «Higher Education in 21st Century: Global cha- 
Tendencias de la Educación universitaria

llenge and national responses», publicado en abril de 1999 por el Institute of International Education. Sin embargo, yo me referiré, principalmente, a las tendencias en Europa, que muestran una fuerte preocupación por el estancamiento de los sistemas universitarios frente a la rápida evolución de la sociedad en los últimos años y la falta de competitividad de las Universidades europeas frente a las norteamericanas.

Partiendo de los documentos e informes mencionados trataré de obtener los conceptos claves que aparecen en todos ellos, conceptos generales que caracterizan las previsiones para la sociedad del siglo XXI y derivando de los mismos las fortalezas y debilidades de las Universidades actuales e indicando los cambios que deben introducirse, pero no solo para adecuarse a los cambios sociales sino para anticiparse e influir en la deriva de los mismos.

\section{Sociedad del Conocimiento}

Esta denominación de la sociedad actual ha calado profundamente en toda la literatura de análisis y previsión social, queriendo enfatizar el hecho de que lo que define el liderazgo y el desarrollo de los países no es ya la producción de bienes materiales y el intercambio de mercancías, sino la generación de conocimientos y su transformación en bienes y servicios, la mayor parte de ellos de carácter inmaterial.

Lo que hoy es un concepto aceptado fue anticipado hace ya casi treinta años, en 1973, por Daniel Bell, profesor de Sociología de la Universidad de Harvard, quién en su libro El advenimiento de la sociedad postindustrial escribía: «La sociedad postindustrial significa, ante todo, un cambio de carácter de la estructura social en una dimensión, y no de la configuración total de la sociedad. Se trata de un tipo ideal, de una construcción acoplada por el analista social, de los diversos cambios de la sociedad que, al reunirlos, resultan mas o menos coherentes contrastados con otras construcciones conceptuales. En la descripción aparecen tres componentes principales: en el sector económico, un giro de la industria a los servicios; en la tecnología, la centralidad de las nuevas industrias basadas en la ciencia; en el terreno sociológico, el crecimiento de nuevas élites técnicas y la introducción de un nuevo tipo de estratificación. Desde aquí, se puede volver atrás y afirmar, de forma más general, que la sociedad postindustrial implica el brote de nuevas estructuras y principios axiales: el paso de una sociedad productora de bienes a una sociedad de información o de conocimiento; y, en los modelos de conocimiento, un cambio del eje de abstracción desde el empi- 
rismo o la chapucería de la prueba y el error a la codificación del conocimiento teórico para dirigir la innovación y la formulación de programas políticos».

La producción de conocimientos, la producción de resultados de la investigación científica, la creación de conocimientos o cualquier otra denominación que quiera darse es una de las funciones de una Universidad moderna y así está formulado en todas las leyes y disposiciones que señalan las funciones o misiones de la Universidad. Como ejemplo, léanse los primeros artículos, tanto de la Ley de Reforma Universitaria (LRU) de 1983, como de la reciente Ley Orgánica de Universidades (LOU), de diciembre de 2001.

Cuando Daniel Bell escribió su libro, en las Universidades españolas se investigaba muy poco. Hoy la realidad es otra: la investigación científica y técnica constituye una parte importante de la labor ordinaria de las Universidades y, aunque no haya alcanzado, en términos cuantitativos, los niveles medios de producción, se puede afirmar que la mayoría del profesorado universitario se aplica a la producción del conocimiento.

Sin embargo, en la actualidad, las Universidades no son las únicas instituciones que producen conocimiento. Nunca, desde su creación en el medievo, lo han sido en exclusiva y en épocas de fuerte crisis de las Universidades, como en el siglo XVIII, gran parte de la creación técnica se realizó al margen de las mismas. Pero es a partir del segundo tercio del siglo XX y, sobre todo, después de la Segunda Guerra Mundial, cuando la investigación científica es abordada de manera sistemática por las empresas y otras instituciones no universitarias, produciendo tanto o mas conocimiento que en las Universidades.

Las Universidades no tienen, pues, la exclusiva en la creación de conocimiento, en la investigación científica y técnica y en la creación artística y es bueno que así sea. Precisamente, la denominación «sociedad del conocimiento" parece indicar también que éste no es exclusivo de ninguna institución y que se ha socializado. La pregunta entonces puede ser: ¿seguirán las Universidades teniendo un papel importante en la producción del conocimiento o se verán relegadas a un segundo plano por otras instituciones?.

La situación se complica más si consideramos, no solo el aspecto de la creación, sino los de almacenamiento y acceso al conocimiento. Las fuentes de información están extendidas por todo el mundo y por muchas instituciones y su acceso a las mismas, con el uso de las tecnologías de la información y de las comunicaciones, es hoy muy sencillo y rápido. En el depósito y acceso a las fuentes del conocimiento también la Universidad ha perdido protagonismo. 


\section{Tendencias de la Educación universitaria}

Si damos un paso más y consideramos la innovación, es decir, la puesta en el mercado de los resultados de la investigación, concepto clave y de honda preocupación en la sociedad, el futuro papel de las Universidades se vuelve a complicar. En efecto, en Europa preocupa mucho lo que se ha dado en llamar «la paradoja de la $\mathrm{I}+\mathrm{D}$, de la investigación y el desarrollo», pues siendo la financiación de la I+D similar a la de Estados Unidos o Japón la innovación tecnológica va detrás de la de estos países, lo cual lleva a pensar que algo no funciona bien en la cadena investigación/desarrollo/sociedad/mercado y convendría pensar en que medida y proporción le corresponde al sistema de enseñanza superior la existencia de esta anomalía, pues hay un consenso en el papel primordial que debe jugar la educación en la sociedad del conocimiento y, dentro de todas las etapas de un sistema educativo, la superior o universitaria es decisiva en el asunto que tratamos.

Señalaba anteriormente que la Universidad no tiene ni mucho menos la exclusiva de la investigación, de la producción de conocimientos y que cada vez más hay otras instituciones de la sociedad que llevan a cabo estas funciones. Observemos, sin embargo, que en las empresas las personas que llevan a cabo tareas de innovación son profesionales formados en las Universidades. Y aquí, en esta función de formación de profesionales, entre los cuales están los investigadores, si que la Universidad tiene prácticamente la exclusiva, lo cual nos lleva a preguntarnos por la importancia de la formación de profesionales para la sociedad del conocimiento.

\section{La formación de profesionales}

La Declaración de Bolonia afirma que: «Está ampliamente reconocido que una Europa del Conocimiento es un factor insustituible para el crecimiento social e individual y un componente indispensable para consolidar y enriquecer la ciudadanía europea, capaz de proporcionar a sus ciudadanos las competencias necesarias para encarar los desafíos del nuevo milenio». Reconocida, pues, la importancia de la educación y de la cooperación educativa, continua diciendo: «Debemos prestar particularmente atención al objetivo de aumentar la competitividad del sistema europeo de educación superior. La vitalidad y eficiencia de una civilización puede medirse por la atracción que su cultura ejerce sobre otros países. Tenemos que asegurar que el sistema europeo de educación superior adquiere un alto grado mundial de atracción que iguale nuestras extraordinarias tradiciones culturales y científicas». Preocupaba a los Ministros de 
Educación europeos, como antes a los de Industria o Innovación, la falta de competitividad del sistema educativo y, en particular, de las Universidades.

En función de estos diagnósticos, la Declaración plantea seis objetivos generales que tienen que ver, principalmente, con la enseñanza, con la formación de profesionales. Así, el primero señala la necesidad de «adoptar un sistema de grados fácilmente entendible y comparable para promover la empleabilidad de los ciudadanos europeos y la competitividad del sistema europeo de educación superior». Y el segundo objetivo marca un sistema cíclico cuando indica que hay que «adoptar un sistema basado en dos ciclos: pregraduado y graduado. El acceso al segundo ciclo requeriría completar con éxito las enseñanzas del primer ciclo que durarán un mínimo de tres años. El grado obtenido después del primer ciclo debe ser relevante para el mercado de trabajo como un nivel apropiado de cualificación. El segundo ciclo debe conducir a los niveles de master y doctorado».

El primer objetivo tiene que ver con otro concepto clave de la actual sociedad: la globalización. Los sistemas educativos de cada país europeo se han quedado pequeños y un tanto cerrados sobre si mismo y hay que buscar una dimensión mayor para competir con otros sistemas, el norteamericano principalmente, más claros y más extensos. El mundo educativo debe seguir el camino emprendido del espacio económico europeo.

El segundo objetivo trata de dar relevancia a la formación de profesionales cualificados para el mercado laboral con el primer grado o título que se obtenga, que debe tener una duración corta. Actualmente, en la mayor parte de los países europeos las enseñanzas se desarrollan de tal modo que los estudiantes obtienen un primer diploma entre los 23 y los 26 años, incluso para carreras cuya duración oficial es de tres años. Las tasas de fracaso son bastante altas y la incorporación al mercado laboral es tardía. Además con el intricado sistema de títulos se producen conflictos de competencias profesionales que a la postre tienen un reflejo en el mundo académico.

La difícil, y por ello nunca bien resuelta, polémica entre formación liberal y formación profesional sigue presente en la concepción de las enseñanzas y los planes de estudio. Encontrar un modelo único para todo tipo de formación es equivocado pero los mensajes que las Universidades reciben de la sociedad son inequívocos en el sentido de reforzar los objetivos de adecuación al mercado laboral. Basta analizar la demanda de estudios por parte de los estudiantes en los últimos diez años en España para comprobar que ésta se dirige hacia las carreras que, según la percepción del estudiante, tienen mayor y mejor proyección en el mercado de 


\section{Tendencias de la Educación universitaria}

trabajo. Las Universidades no pueden mirar hacia otro lado, pensando que la sociedad se equivoca y seguir amparándose en una libertad académica y en una autonomía mal entendidas.

\section{Creatividad, enseñanza y aprendizaje}

Recordando el poco éxito de la producción o creación de conocimientos y la escasa innovación tecnológica de la sociedad europea frente a la norteamericana, cabe pensar si en alguna medida la formación de profesionales tiene que ver con ello. Yo pienso que sí. La formación actual está centrada principalmente en el desarrollo de contenidos disciplinares, en asignaturas diseñadas por los profesores en función, no solo pero si de manera importante, de sus puntos de vista y, lo que es peor, de sus intereses. El resultado es una acumulación de contenidos, unos curricula muy densos que convierten el trabajo del estudiante en un ejercicio de repetición de lo explicado por el profesor dejando apenas espacio y tiempo para el desarrollo de su creatividad. La creatividad no es una disciplina, una asignatura que pueda ser explicada en el aula. La creatividad no se enseña, se aprende, y depende de las condiciones en que se desarrolla la enseñanza. La creación, además de libertad, que ya se tiene, requiere reflexión y ésta requiere tiempo, lo cual debe llevar a las Universidades a prestar más atención al fomento de la creatividad en los estudiantes mediante la innovación en los contenidos curriculares de las enseñanzas y en los métodos de concebir la formación.

En un mundo que experimenta cambios rápidos, se percibe la necesidad de una nueva visión y un nuevo paradigma de la enseñanza superior, que tendrá que estar más orientadas al estudiante. Es lo que se denomina de manera esquemática el cambio de énfasis de la enseñanza al aprendizaje. Como señala la Declaración de la conferencia Mundial de la UNESCO de octubre de 1998: «Para conseguir estos objetivos es posible que haya que reestructurar los curricula, implantando métodos nuevos y adecuados que vayan más allá del dominio cognoscitivo de las disciplinas. Se han de fomentar nuevos puntos de vista pedagógicos y didácticos para facilitar la adquisición de técnicas, competencias y capacidades de comunicación, creatividad y análisis crítico, pensamiento independiente y trabajo en equipo en contextos multiculturales, donde la creatividad también implica combinar el saber y los conocimientos locales y tradiciones con la ciencia y las tecnologías avanzadas».

Una de las claves, pues, del estancamiento de la innovación en Europa está en el proceso formativo de los graduados superiores. El proceso 
cíclico marcado por el segundo objetivo de la Declaración de Bolonia parece buscar el necesario equilibrio entre la formación creativa orientada al mercado, la especialización concreta y la formación de investigadores. El medio plazo lo fijarían las tendencias y la evolución del mercado y se cubrirían con un primer grado suficiente poco especializado donde el método fuera tal que se fomentará el aprendizaje y la creatividad; el corto plazo se desarrollaría mediante el grado de master, flexible y cambiable según las demandas del mercado; y el largo plazo se haría mediante la formación de doctores, de investigadores donde la creatividad y la producción de conocimientos sería lo fundamental. Es en suma, aunque cueste reconocerlo como tal, el modelo de las mejores universidades norteamericanas.

\section{Movilidad, internacionalización y cooperación}

Todo lo anterior debe hacerse teniendo en cuenta otra de las características que definen la sociedad del siglo XXI y que ya se mencionó anteriormente: la globalización. A ello apuntan los cuatro objetivos siguientes a los dos analizados, de la Declaración de Bolonia, cuando establece: «La promoción de la movilidad removiendo los obstáculos para el ejercicio efectivo del libre movimiento de los estudiantes y de los profesores y del personal de administración, reconociendo y valorando los periodos atendidos en otras Universidades realizando tareas de investigación, enseñanza y aprendizaje». O cuando habla de «La promoción de la dimensión europea de la educación superior, particularmente en el desarrollo de los curricula, la cooperación interinstitucional, esquemas de movilidad y programas integrados de estudio, aprendizaje e investigación».

Para facilitar estos objetivos la Declaración de Bolonia propone: «La adopción de un sistema de créditos -tal como el sistema ECTS- como un medio adecuado para promover la movilidad estudiantil lo más amplia posible, considerando que los créditos pueden obtenerse en contextos no universitarios, incluyendo la formación durante toda la vida». El sistema ECTS -European Credit Transference System- ha sido ensayado con éxito relativo en el programa ERASMUS de movilidad de estudiantes. Se trata de un sistema de valoración de las enseñanzas basado en el esfuerzo y el trabajo del estudiante, es decir, en el aprendizaje, más que en la carga horaria lectiva, basada en las horas de clase del profesor. Créditos que deben ser transferibles entre instituciones y acumulables para la obtención de los diversos grados. Para ello, la unidad de medida, el crédito, 


\section{Tendencias de la Educación universitaria}

debe ser lo más homogénea, cuantitativa y cualitativamente, en todos los países europeos.

Un ejemplo nos ayudará a entender la importancia del establecimiento del sistema ECTS. Si se parte de que la jornada laboral del estudiante es de 1800 horas al año y se define el crédito como el equivalente a 30 horas de trabajo un curso se compondría de 60 créditos. Si se estima que el número de semanas efectivas de trabajo, incluyendo exámenes y periodos de prácticas, es de 40 semanas esto implica una media de 1,5 créditos por semana. Compárese esto con el caso español con una media de 2,5 créditos -25 horas de clase- por semana para comprender que debe hacerse un esfuerzo por reducir los contenidos, la explicación clásica en las clases presenciales, y aumentar la labor de orientación, de tutoría, de fomento y valoración de trabajo personal y en equipo de los estudiantes. Esto requiere un cambio de cultura y de hábitos del profesorado, que, experiencias recientes, muestran que es muy difícil. Permítanme que cuente mi experiencia personal. En mi etapa de estudiante en la Universidad Politécnica de Madrid el número de horas de clase rondaba las 25 semanales y el esfuerzo para aprobar era notable. En mis estudios de postgrado en una prestigiosa universidad de California las horas de clase no pasaban de 12 a la semana y sin embargo mi esfuerzo personal era mayor que el que tuve que hacer en España. Esto es el método basado en el aprendizaje, distinto al basado en la enseñanza.

En lo referente a la cooperación institucional -incluida la cooperación con el mundo empresarial y las administraciones públicas además de con otras universidades- la movilidad y la innovación, el ejemplo de la gestación, desarrollo e implantación de INTERNET es un modelo paradigmático de un éxito sin precedentes del modo de hacer de las universidades norteamericanas. Queda fuera del contenido de este artículo relatar esa historia reciente pero recomiendo estudiarla en la amplia bibliografía existente, entre la cual citaré el libro, recientemente publicado, «La Galaxia de INTERNET» del profesor y sociólogo español Manuel Castell, de cuyo libro tomaré las siguientes citas: «Así pues, la cultura de Internet radica en la tradición académica de la investigación científica compartida, la reputación obtenida gracias al prestigio académico, la evaluación por parte de los colegas y la apertura y publicidad de las investigaciones. Históricamente, Internet se construyó en los círculos, tanto en las atalayas de los catedráticos, como en las trincheras de los estudiantes de doctorado, cuyos valores, hábitos y conocimientos se transmitieron a la cultura hacker». El papel de las universidades, la cooperación entre ellas, la movilidad del personal entre las universidades, 
los centros de investigación de grandes empresas y la cooperación y financiación de departamentos de la administración USA muestran un ejemplo de los valores que estamos señalando. En otra página del libro escribe: «Si existe una idea compartida sobre las consecuencia sociales del creciente acceso a la información es que la educación y el aprendizaje a lo largo de la vida constituyen herramientas esenciales para el éxito en el trabajo y el desarrollo personal. Lo fundamental es cambiar el concepto de aprender por el de aprender a aprender (...) En otras palabras, el nuevo aprendizaje está orientada hacia el desarrollo de la capacidad educativa que permite transformar la información en conocimiento y el conocimiento en acción».

\section{Nuevos métodos, nuevas tecnologías, nuevas demandas}

La alusión a Internet como ejemplo de creatividad y cooperación institucional llevado a cabo por Universidades norteamericanas nos conduce directamente a la importancia creciente de las Tecnologías de la Información y de las Comunicaciones (TIC) en todas las fases y ciclos de la enseñanza superior. Empieza a ser un tópico manejado en todos los informes pero, si la sociedad del conocimiento ha sido posible gracias a estas tecnologías que alteran notablemente las formas de producción, de almacenamiento, acceso y difusión de la información, parece claro que las Universidades tendrán que utilizarlas de forma sistemática en sus funciones de creación, transmisión y almacenamiento de la información y en sus procesos de investigación y formación de profesionales. A pesar de ello, las Universidades se muestran muy lentas en la adaptación de sus métodos, normas y formas organizacionales a esta nueva realidad mientras que otras instituciones no universitarias se muestran muy activas en segmentos de la educación donde las legislaciones nacionales les permiten operar sin restricciones.

La Conferencia de Rectores de las Universidades Europeas (CRE) lanzó en 1996 un proyecto para explorar el impacto de las TIC en la Universidad y publicó, posteriormente, un documento titulado «Reestructurando la Universidad: el desafío de las nuevas tecnologías». En dicho documento se recogen, entre otras muchas las siguientes conclusiones: falta de estrategias institucionales claras para suministrar un marco de trabajo para el desarrollo y utilización de las nuevas tecnologías en la enseñanza; fuerte resistencia tanto de los profesores como del personal de administración para usar estas tecnologías; problemas para estimar los costes de estas actividades, que son a menudo infraestimadas. Los pro- 


\section{Tendencias de la Educación universitaria}

pios Rectores reconocen que se avanza poco al tiempo que intuyen que es necesario dar pasos rápidos hacia delante.

No se trata tan solo de un uso meramente instrumental de estas tecnologías en los procesos docentes, como cambiar la pizarra y la tiza por el cañón de vídeo o el programa «Power point», o enviar documentos en formato clásico vía Internet. Se trata de cambiar las formas de organización de la enseñanza, desde la matriculación y seguimiento de los estudiantes, la preparación y presentación de materiales, la tutoría permanente, el concepto de horario y de tareas, el coste de las enseñanzas, la valoración y acreditación de las mismas, las condiciones para la obtención de un grado y otras muchas mas. No se trata solo de crear Universidades abiertas, o a distancia, o virtuales, sino que todas las Universidades combinen procesos presenciales con procesos a distancia usando las tecnologías de la información y de las comunicaciones con nuevos métodos apropiados al uso de tan poderosa herramienta.

A todo lo anterior se une la necesidad de atender una creciente demanda de formación superior de individuos y grupos que no son las cohortes tradicionales de bachilleres de 18 años procedentes, principalmente, del entorno geográfico próximo al campus universitario. En efecto, en los últimos años no solo se ha estancado el número de estudiantes que solicitan ingresar en la Universidad, sino que está disminuyendo a un fuerte ritmo que continuará, por lo menos, hasta que los hijos de la creciente población inmigrante llame a las puertas de la Universidad. Este fenómeno, reciente en España, junto con el aumento del número de centros universitarios ha empezado a preocupar seriamente a las Universidades que, lenta y reactivamente, comienzan a modificar sus actitudes de contención de la demanda a realizar campañas de información para atraer estudiantes a sus aulas.

Sin embargo, otros tipos de demanda están creciendo. La más importante es la denominada formación continua, formación posgraduada o formación durante toda la vida («long life learning»). Se acabó ya hace años el concepto de que una vez obtenida la graduación en una Universidad solo había que dejar correr a la experiencia en el puesto de trabajo. Aprender con la experiencia sigue siendo obvio pero, además, en todas las profesiones basadas en el conocimiento la necesidad de la actualización es imperiosa. Hay un gran mercado de la formación continua en el que participan muchas empresas de sectores no educativos o empresas educativas surgidas de empresas industriales o comerciales que realizan su actividad y su negocio en este gran mercado. Las Universidades también han entrado en este mercado, en parte para obtener recursos financieros adicionales, pero me temo que la mayor parte del mercado no está en las Universidades. Ya señale anteriormente 


\section{Vicente Ortega Castro}

que la investigación y el desarrollo no eran exclusivas de las Universidades. Tampoco lo es, ni debe serlo, la formación continua y ésta tiene una demanda creciente en la que las Universidades tienen que competir con otras instituciones muchas veces más adaptadas a procesos competitivos, con formas de organización adecuadas al tipo de demanda y con estructuras más ágiles y flexibles que las que poseen las Universidades que no acaban de integrar de forma eficaz este tipo de formación dentro de sus estructuras organizativas tradicionales sin establecer por ello nuevas estructuras.

Otro tipo de demanda, aun incipiente, pero que puede aumentar en el futuro próximo sobre todo si se hace una oferta atractiva, es la derivada del aumento de la población de jubilados que buscan una oferta de tipo cultural para llenar tiempo de ocio, satisfacer anhelos no satisfechos en su edad joven u otras causas. Evidentemente, los métodos y los contenidos de los estudios no pueden ser los mismos que los empleados para los bachilleres.

Y por último otro tipo de demanda lo constituye el contingente de estudiantes extranjeros tanto en los ciclos de pregraduación como en los de graduación. En España, el porcentaje de alumnos extranjeros en las Universidades apenas alcanza el $0,5 \%$ en los estudios pregraduados y el $8 \%$ en posgrado y doctorado, cifras ridículas si las comparamos con las existentes en muchas Universidades norteamericanas. Ya vimos al comentar la Declaración de Bolonia la preocupación existente en Europa por la falta de competitividad del sistema europeo de educación superior en el mercado global de dicha educación.

La educación superior, factor de suma importancia en la sociedad del conocimiento, es por ello mismo un sector con implicaciones económicas y sociales en el desarrollo de una sociedad que se rige por reglas de mercado, hasta tal punto que empieza a ser considerada como sector a regular por la Organización Mundial del Comercio. Esto no quiere decir que las Universidades tengan que regirse por reglas de mercado pero tampoco puede aislarse y orientar sus actuaciones fuera de las grandes tendencias sociales. Es necesario que emprenda nuevo métodos basados en el aprendizaje con la incorporación de las tecnologías de la información y de las comunicaciones y que cambie sus esquemas, tradicionales de organización de la enseñanza y de la investigación teniendo en cuenta las nuevas demandas y las nuevas necesidades en un mundo globalizado y competitivo.

\section{Calidad y rendición de cuentas}

Hasta la década de los noventa en Europa no preocupaba demasiado la promoción y la medición de la calidad en las Universidades. Con muy 


\section{Tendencias de la Educación universitaria}

poca tradición en procedimientos de evaluación y acreditación de las enseñanzas, la investigación y los resultados de los procesos, la única calidad percibida era la resultante de la antigüedad o de la dimensión de la institución universitaria. Situación ésta muy distinta de la existente en Norteamérica, donde instituciones externas a las Universidades, incluida la prensa especializada, se ocupan de evaluar las actividades de las Universidades, acreditar sus programas y grados y establecer, incluso, clasificaciones para informar a los ciudadanos a la hora de tomar decisiones.

Las Universidades tienen, y deben tener, un alto grado de autonomía de organización, de gobierno y de gestión. Son, en su mayoría, instituciones públicas financiadas en más de tres cuartas partes por presupuestos de las Administraciones Públicas, es decir, con los impuestos de los ciudadanos. El propio concepto de «servicio público de la educación superior» lleva a que la contrapartida a la autonomía sea el correcto ejercicio de su responsabilidad social, para que la Universidad pública pueda rendir a la sociedad lo que ésta tiene derecho a exigirle: calidad docente e investigadora y utilización eficaz y eficiente de los recursos públicos puestos a su disposición.

A su vez, los gobiernos democráticos tienen la obligación de rendir cuentas a los Parlamentos de sus políticas y de la utilización de sus presupuestos. El interés que tienen los procesos de evaluación y acreditación para la sociedad es claro y se debe centrar en un triple aspecto: informar a la sociedad (familias, empresas e instituciones) para una mejor toda de decisiones; orientar las políticas de asignación de recursos públicos y estimular a las propias Universidades en sus procesos de permanente perfeccionamiento y mejora.

Decía que en Europa, siguiendo una vez mas el modelo de Estados Unidos, la preocupación por los temas de evaluación de la calidad dan paso a la acción en 1991, cuando se elevó a consideración una propuesta de recomendación del Consejo, relativa a la «cooperación europea en materia de garantía de la calidad en la enseñanza superior. En España, después de un «Programa experimental de evaluación del sistema universitario» puesto en marcha por el Consejo de Universidades en 1992 y de la participación en el «Proyecto Piloto europeo de evaluación de la calidad de la enseñanza superior» en el bienio 1994-95, se puso en marcha el Plan Nacional de Evaluación de la Calidad de las Universidades que, actualmente, y con modificaciones, continua desarrollándose.

En Europa ha ido calando el concepto y las prácticas de evaluación de la calidad de modo que la Declaración de Bolonia, ya citada anteriormente, recoge explícitamente la importancia de la medición de la calidad 
cuando en el punto quinto recomienda: «La promoción de la cooperación Europea en la garantía de la calidad con vistas a desarrollar criterios y metodologías comparables».

Así pues, la medida del equilibro entre la autonomía universitaria y su responsabilidad social a través de, entre otros factores, la evaluación, la acreditación y la certificación de la calidad se revela como una de las tendencias del nuevo milenio y cobra mayor importancia, en el nuevo mercado de la educación superior, en el que las Universidades tendrán que competir entre ellas y con otras instituciones docentes e investigadoras.

\section{Complejidad, multifuncionalidad y financiación}

En el último cuarto de siglo se ha pasado de una Universidad de élites a la Universidad de masas. El crecimiento de casi todos los parámetros de los sistemas universitarios: número de estudiantes, número de centros, número de graduados, etc..., ha sido espectacular. También la financiación pública ha crecido pero en menor proporción que los otros crecimientos y, últimamente, tiende a estancarse. Ahora que la demanda tradicional de enseñanza, la de los bachilleres, tiende a disminuir, nuevas demanda educación superior surgen.

La sociedad pide a las Universidades que forme profesionales válidos para el mercado laboral; que forme individuos creativos e innovadores; que cree conocimientos a través de una investigación que sea a la vez básica y orientada a las necesidades de la sociedad; que atienda nuevas demandas de formación durante toda la vida; que se internacionalice y que sus profesores y estudiantes se muevan entre universidades y empresas y cooperen con otras instituciones sociales; que lo hagan todo con unos estándares altos de calidad, rindiendo cuentas a la sociedad; y todo ello dentro de un mercado cada vez mas globalizado, mas abierto y más competitivo donde la creación de conocimientos y la formación ha dejado de ser exclusiva de las Universidades. Es decir, a las funciones clásicas de las Universidades se les añaden otras nuevas y se demandan nuevos métodos para llevar a cabo las antiguas. En suma, las Universidades han de desarrollar sus funciones en un panorama complejo y múltiple con esquemas de multifuncionalidad difíciles de implementar por falta de una cultura organizacional adecuada a estas múltiples tareas que, a veces, parecen incluso contradictorias.

A las dificultades intrínsecas de esta complejidad y de esta multifuncionalidad se suma la extrínseca de un estancamiento en las formas tra- 


\section{Tendencias de la Educación universitaria}

dicionales de financiación pública y de la exigencia de que sean cada vez más importantes los recursos obtenidos del sector privado, sea por un aumento de los precios públicos a los estudiantes, por servicios de investigación al mundo empresarial o por otras prestaciones de servicios. Es lo que algunos movimientos estudiantiles han llamado exagerada e impropiamente la privatización de la Universidad.

Se ha llegado a discutir el concepto tan arraigado en Europa de la enseñanza superior como servicio público y en todos los países se discuten los problemas de equidad ligados a quién debe pagar el coste del servicio. Temas de honda raíz política que seguirán dando que hablar en las próximas décadas. Pero una tendencia parece evidente, independiente del color más liberal o más social de los gobiernos de turno: la financiación pública ha alcanzado, o está a punto de alcanzar, su techo y los esquemas clásicos de financiación indiscriminada tienden a ser sustituidos por políticas de financiación basadas en programas competitivos y en los resultados obtenidos. Dicho de otro modo, la financiación pública crecerá poco y será más exigente.

En este panorama, que tiene aspectos positivos y negativos, las Universidades tendrán que diseñar estrategias de búsqueda y de gestión de recursos para la institución como un todo, combinando políticas de incentivo para los departamentos y profesores más competentes, con políticas de subsidio cruzado para la Universidad en su conjunto. Las Universidades, dentro de la multiplicidad de funciones que la caracterizan tendrán que examinar sus fortalezas y otear sus oportunidades y ajustar sus actividades en función de ellas y de sus recursos. No todos las Universidades podrán abordar con éxito todas las funciones y se producirá inevitablemente una diferenciación entre Universidades, circunstancia que en Europa empieza a producirse pero que en los Estados Unidos es moneda corriente desde hace mucho tiempo.

\section{Conclusión}

Las nuevas formas de creación, almacenamiento y transmisión de la información del conocimiento, y de formación de profesionales en un mundo globalizado, más desregulado y más competitivo en un escenario de nuevas formas de financiación plantea desafíos a las Universidades que deben ser aprovechados haciendo un esfuerzo de imaginación para actuar proactivamente ante la situación y no reactivamente como lo han hecho en ocasiones anteriores. Como decía Albert Einstein: «en épocas de crisis solo la imaginación es más importante que el conocimiento». 